5 Sean Thomas ${ }^{1 *}$, Kathryn Wierenga ${ }^{2,3 *}$, James Pestka ${ }^{3,4,5}$, Andrew J Olive $^{1}$

\title{
8 Affiliations:
}

9 1. Department of Microbiology and Molecular Genetics, College of Osteopathic Medicine, 10 Michigan State University, East Lansing MI USA.

11 2. Department of Biochemistry and Molecular Biology, Michigan State University, East Lansing, 12 MI 48824, U.S.

13 3. Institute for Integrative Toxicology, Michigan State University, East Lansing, MI 48824, U.S.

14 4. Department of Microbiology and Molecular Genetics, Michigan State University, East Lansing, 15 MI 48824, U.S.

16 5. Department of Food Science and Human Nutrition, East Lansing, MI 48824, U.S.

17 *These authors contributed equally to this work.

19 Short-Title: Functional genetics in AM-like cells.

\section{Corresponding Author:}

22 Andrew Olive Ph.D.

23 Department of Microbiology and Molecular Genetics

24 College of Osteopathic Medicine, Michigan State University

25 East Lansing, MI 48824, U.S.

26 Email: oliveand@msu.edu 


\section{KEYWORDS}

31 Macrophage, lung, silica, CRISPR, TGF $\beta$, Siglec-F, IL-1, MARCO, peroxisomes

\section{ABSTRACT}

34 Alveolar macrophages (AMs) are tissue resident cells in the lungs derived from the fetal liver

35 that maintain lung homeostasis and respond to inhaled stimuli. While the importance of AMs is

36 undisputed, they remain refractory to standard experimental approaches and high-throughput

37 functional genetics as they are challenging to isolate and rapidly lose AM properties in standard

38 culture. This limitation hinders our understanding of key regulatory mechanisms that control

39 AM maintenance and function. Here, we describe the development of a new model, fetal liver-

40 derived alveolar-like macrophages (FLAMs), which maintains cellular morphologies, expression

41 profiles, and functional mechanisms similar to murine AMs. FLAMs combine treatment with

42 two key cytokines for AM maintenance, GM-CSF and TGF $\beta$. We leveraged the long-term

43 stability of FLAMs to develop functional genetic tools using CRISPR-Cas9-mediated gene editing.

44 Targeted editing confirmed the role of AM-specific gene Marco and the IL-1 receptor //1r1 in

45 modulating the AM response to crystalline silica. Furthermore, a genome-wide knockout library

46 using FLAMs identified novel genes required for surface expression of the AM marker Siglec-F,

47 most notably those related to the peroxisome. Taken together, our results suggest that FLAMs

48 are a stable, self-replicating model of AM function that enables previously impossible global

49 genetic approaches to define the underlying mechanisms of AM maintenance and function.

50

51 


\section{INTRODUCTION}

53 Tissue resident immune cells regulate homeostasis and control local inflammation to external

54 stimuli. A subset of these immune cells are tissue resident macrophages (TRMs) that sample the

55 environment and initiate host responses (1). Distinct TRM populations exist in specific tissues

56 including the liver (Kupffer cells), the skin (Langerhans cells), the brain (microglia), and the lungs

57 (alveolar macrophages [AMs]). These distinct TRMs all have unique functions that are regulated

58 by the local environment and are required for tissue maintenance $(2,3)$.

As the first line of defense in the airways, AMs are particularly important for tuning the host

61 immune response in the lungs (4). AMs can be distinguished from other macrophage

62 populations in the lung by the surface expression of the sialic acid receptor Siglec- $F$, the

63 scavenger receptor MARCO, and the integrin CD11C in addition to the high expression and

64 activity of the transcription factor PPAR $\gamma$, which drives many AM-specific genes $(5,6)$. AMs are a

65 long-lived and self-replicating and, like most TRMs, are derived from embryonic precursors (7).

66 AMs arise from fetal liver monocytes, which migrate to the lung and develop into mature AMs

67 in the presence of cytokines such as GM-CSF and TGF $\beta$ shortly after birth (8-10). The continued

68 presence of these factors is necessary for the maintenance and self-renewal of AMs in the lung,

69 in part by promoting expression and activation of $\operatorname{PPAR} \gamma(10,11)$. Genes and pathways induced

70 by this receptor are involved in lipid metabolism and induction of scavenger receptors that

71 promote phagocytosis (12). This is critical for the AM roles of maintaining surfactant

72 homeostasis, efferocytosis of cellular debris, and phagocytosis of inhaled microbes and

73 particles in the alveolar space $(12,13)$. Impaired clearance of surfactant by AMs can result in

74 the pathophysiological condition known as pulmonary alveolar proteinosis (PAP) (14). In

75 addition, reduced AM efferocytosis and phagocytosis has been observed in patients with

76 asthma, COPD, and cystic fibrosis, likely contributing to the sustained inflammation and

77 susceptibility to infection observed in these diseases (15-19).

79 Despite the paramount importance of AMs for lung health, there continue to be key gaps in our 80 understanding of how they are maintained and function to regulate the host response in the 
81 lungs. One hurdle towards a mechanistic understanding of AMs is that experiments employing

82 primary AMs require large numbers of animals to isolate a small number of cells that do not

83 robustly proliferate or maintain AM-like functions ex vivo (20). This limitation has prevented

84 genetic approaches from being employed to better understand AM maintenance and function.

85 As a result, many ex vivo studies investigating responses to airborne particles and microbes rely

86 on bone-marrow derived macrophages (BMDMs) or transformed macrophage cell lines as

87 surrogates of AM biology (21-23). While these macrophage models are useful, they do not

88 faithfully recapitulate all AM functions (24-26). A recent alternative approach cultured cells

89 from the murine fetal liver in the presence of GM-CSF to generate AM-like cells that are

90 functionally and phenotypically like AMs (25). This approach enabled the isolation of large

91 numbers of AM-like cells that might be amenable to tractable genetic approaches. However, we

92 found here that fetal liver-derived cells cultured in GM-CSF alone lost their AM-like

93 morphology, phenotype, and surface marker expression over time, suggesting that GM-CSF is

94 insufficient to maintain the AM-like phenotype. A recent study found that AMs could be

95 continuously cultured ex vivo in the presence of GM-CSF and TGF $\beta$ (27), which is consistent

96 with reports that TGF $\beta$ promotes AM development and maintains AM function both in vivo and

97 ex vivo (10).

99 Here, we found that growing fetal liver cells in both GM-CSF and TGF $\beta$ results in a long-term

100 stable population of cells that are phenotypically and functionally similar to AMs. Using these

101 fetal liver-derived alveolar-like macrophages (FLAMs), we developed targeted and global

102 genetic tools to dissect regulatory networks that are required to maintain AM-like cells and

103 function. Employing targeted gene-editing, we show here that directed mutations are readily

104 introduced in FLAMs to query specific AM functions. We further demonstrate the utility of

105 FLAMs by using genome-wide CRISPR-Cas9 knockout screen to identify genes that are required

106 for the surface expression of the AM-specific marker Siglec-F. The screen identified key

107 pathways used to maintain Siglec-F expression and the AM-like state including the observation

108 that peroxisome biogenesis plays a central role in maintaining AM functions. Together our 
results show that FLAMs enable the global dissection of AM regulatory mechanisms at a previously impossible scale.

\section{MATERIALS AND METHODS}

\section{Animals}

114 Experimental protocols were approved by the Institutional Animal Care and Use Committee at 115 MSU (AUF \# PROTO201800113). 6-8 week old C57BI6 mice (cat \# 000664) and Cas9 ${ }^{+}$mice (cat \# 116 026179) were obtained from Jackson Laboratories (Bar Harbor, ME). Mice were given free

117 access to food and water under controlled conditions (humidity: 40-55\%; lighting: 12 hour

118 light/dark cycles; and temperature: $\left.24 \pm 2^{\circ} \mathrm{C}\right)$ as described previously $(28,29)$. Pregnant dams at

119 8-10 weeks of age and 14-18 gestational days were euthanized to obtain murine fetuses. AMs

120 were isolated from male and female mice older than 10 weeks of age. BMDMs were obtained

121 from male and female mice 6 weeks of age and older.

\section{FLAMs cell isolation and culture}

124 Fetal liver derived cells were obtained as previously described (30). Briefly, pregnant dams were 125 euthanized by $\mathrm{CO}_{2}$ inhalation for $10 \mathrm{~min}$ to ensure death to neonates, which are resistant to 126 anoxia. Cervical dislocation was used as a secondary form of death for the dam. Fetuses were 127 immediately removed, and loss of maternal blood supply served as a secondary form of death

128 for the fetuses. Cells were cultured in complete Roswell Park Memorial Institute medium 129 (RPMI, Thermo Fisher) containing 10\% fetal bovine serum (FBS, R\&D Systems), 1\% penicillin130 streptomycin (P/S, Thermo Fisher), 30 ng/mL recombinant mGM-CSF (Peprotech), and 20 $131 \mathrm{ng} / \mathrm{mL}$ recombinant hTGF $\beta 1$ (Peprotech) included where indicated. Media was refreshed every 132 2-3 days. When cells reached 70-90\% confluency, they were lifted by incubating for 10 minutes 133 with $37^{\circ} \mathrm{C}$ phosphate-buffered saline (PBS) containing $10 \mathrm{mM}$ EDTA, followed by gentle 134 scraping. After approximately 1 week, adherent cells adopted a round, AM-like morphology. At 135 this time, stocks were frozen for future use. Thawed stocks were plated in untreated Petri 136 dishes with either GM-CSF or GM-CSF and TGF $\beta$ (20 ng/mL recombinant hTGF $\beta 1$ [Peprotech]) 137 and sub-cultured as described above. 
AM isolation and culture

140 Mice were euthanized by $\mathrm{CO}_{2}$ exposure followed by exsanguination via the inferior vena cava.

141 Lungs were lavaged as previously described (20). Cells were then resuspended in RPMI media

142 containing $30 \mathrm{ng} / \mathrm{mL}$ GM-CSF and plated in untreated 48- or 24- well plates. AMs were lifted

143 from plates using Accutase ${ }^{\mathrm{TM}}$ (BioLegend) and seeded for experiments.

\section{BMDM isolation and culture}

$146 \mathrm{C} 57 \mathrm{BL} / 6 \mathrm{~J}$ mice were euthanized by $\mathrm{CO}_{2}$ exposure followed by cervical dislocation. Both femurs

147 were cut on one end to expose the bone marrow, placed cut side down in $0.6 \mathrm{~mL}$ tubes, and

148 centrifuged at 16,000 xg for 25 seconds. Marrow from multiple mice was pooled, dissociated to

149 a single cells suspension in sterile PBS and pelleted by centrifuging at $220 \mathrm{xg}$ for $5 \mathrm{~min}$. The

150 pellet was resuspended in mouse RBC lysis buffer (Alfa Aeser) and incubated at room

151 temperature for 5 minutes. The RBC lysis buffer was diluted with 2 volumes of PBS and the cell

152 suspension passed through a nylon $70 \mu \mathrm{m}$ filter (Corning). Cells were pelleted a second time

153 and resuspended in RPMI media containing 10\% FBS, 1\% P/S, and 20\% L929 media (31).

154 Approximately $5 \times 10^{6}$ cells were plated per dish in $10 \mathrm{~cm}$ untreated petri dishes. Media was

155 refreshed every 2-3 days. Cells were used for assays when fully differentiated after 7 days.

\section{Flow cytometry}

158 Plated cells were lifted in warm PBS with 10 mM EDTA for 5-10 minutes and washed twice in

159 PBS before fluorescent antibody labeling. Immediately following isolation, AMs were

160 resuspended in PBS and filtered through a $70 \mu \mathrm{m}$ basket filter and incubated with an antibody

161 cocktail of PE CD170, APC CD11C, APC-Cyanine7 CD14, and FC Block (Biolegend; 1:400 in PBS)

162 for $20 \mathrm{~min}$ at room temperature in light-free condition. Immunochemically labeled cells were

163 washed three times with PBS, resuspended in PBS, and passed through a $70 \mu \mathrm{m}$ nylon filter

164 immediately prior to analysis. Flow cytometry was performed on a LSR II Flow Cytometer (BD

165 Biosciences) at the Michigan State University Flow Cytometry Core. 


\section{$167 q P C R$}

168 RNA was isolated from $\sim 5 \times 10^{5}$ cells using RNeasy mini kits (Qiagen), typically yielding 100-400

169 ng RNA. RNA was then reverse transcribed to cDNA using a High-Capacity cDNA reverse

170 transcription kit (Thermo Fisher) on a Stratagene Robocycler 40. Quantitative real-time qPCR

171 was performed using specific Taqman probes (Thermo Fisher) for TGF $\beta 1$ (Tgfb1), TGF $\beta$

172 receptors (Tgfbr1, Tgrbr1), selected genes used to distinguish AMs from other macrophage

173 populations (Cd14, Siglecf, Marco, Pparg Car4, Fabp4, Itgax), and cytokines (II1a, I/1b, I/10) on

174 an Applied Biosystems ${ }^{\text {TM }}$ QuantStudio $^{\text {TM }} 7$ real-time PCR system. Data were analyzed with

175 Applied Biosystems ${ }^{\mathrm{TM}}$ Thermo Fisher Cloud using the RQ software and the relative

176 quantification method. Gapdh was used as the housekeeping gene. Relative copy number (RCN)

177 for each gene was normalized to expression of Gapdh and calculated as described previously

178 (32).

Scanning electron microscopy

181 Suspensions of AMs or FLAMs were diluted to $2.5 \times 10^{5}$ cells $/ \mathrm{mL}$, and $100 \mu \mathrm{L}$ pipetted directly

182 upon glass $12 \mathrm{~mm}$ diameter, 0.13-0.16 mm thick circular coverslips (Electron Microscopy

183 Sciences), which were placed in the bottom of 6-well plates. Cells were allowed to settle for 2-3

184 minutes, then $1 \mathrm{~mL}$ of media was added to fill the well. To fix cells, the coverslips were removed

185 from the wells, submerged in $4 \%$ glutaraldehyde in $0.1 \mathrm{M}$ sodium phosphate buffer at $\mathrm{pH} 7.4$

186 and placed in a graded ethanol series (25\%, 50\%, 75\%, 95\%) for $10 \mathrm{~min}$ at each step followed by

1873 minutes changes in $100 \%$ ethanol.

189 Samples were critical point dried in a Leica Microsystems model EM CPD300 critical point drier

190 (Leica Microsystems, Vienna, Austria) using $\mathrm{CO}_{2}$ as the transitional fluid. Coverslips were then

191 mounted on aluminum stubs using epoxy glue (System's Three Quick Cure 5, Systems Three

192 Resins, Auburn WA). Samples were coated with osmium at $10 \mathrm{~nm}$ thickness in an NEOC-AT

193 osmium chemical vapor deposition coater (Meiwafosis Co, Osaka, Japan) and examined in a

194 JEOL 7500F (field emission emitter) scanning electron microscope (JEOL, Tokyo, Japan). 


\section{$\mathrm{CSiO}_{2}$ phagocytosis assay}

197 To assess phagocytosis of $\mathrm{cSiO}_{2}$ particles, FLAMs, AMs, and BMDMs were seeded at 0.25

198 cells $/ \mathrm{cm}^{2}$ in 48- or 96 -well plates to observe engulfment of surrounding silica particles. The

199 following day, the media was removed, wells were rinsed $1 x$ with sterile PBS, media replaced

200 with FluoroBrite DMEM (Thermo Fisher) containing 10\% FBS and 200 nM SYTOX Green nucleic

201 acid stain (Thermo Fisher). $\mathrm{CSiO}_{2}$ was then added dropwise to a final density of $25-100 \mu \mathrm{g} / \mathrm{cm}_{2}$.

202 Cells were imaged over time on an EVOS FL2 fluorescent microscope (Thermo Fisher) with an

203 on-stage, temperature control $\mathrm{CO}_{2}$ incubator and 2-4 images were acquired per well. SYTOX

204 Green detected on the GFP light cube.

205

206 Images were analyzed using analysis pipelines built in the CellProfiler software (33). $\mathrm{CSiO}_{2}$

207 engulfment was assessed by quantifying the number of $\mathrm{cSiO}_{2}$-filled cells, which have a higher

208 pixel intensity than non- $\mathrm{CSiO}_{2}$-filled cells due to the accumulation of the particles. To avoid

209 counting aggregated $\mathrm{cSiO}_{2}$ particles, a threshold was applied to capture only shapes with high

210 solidity and low compactness. Cell death was quantified by counting SYTOX Green ${ }^{+}$cells,

211 respectively.

213 ELISAS

214 Cells were treated with $\mathrm{CSiO}_{2}$ for 8 hours or LPS for 24 hours at the indicated concentrations.

215 Cell-free supernatant was collected and the cytokines IL-1 $\alpha$, IL-1 $\beta$, and IL-10 were analyzed

216 using DuoSet ELISA kits (R\&D Systems) per the manufacturer's instructions.

\section{CRISPR Targeted Knockouts}

219 sgRNA cloning sgOpti was a gift from Eric Lander \& David Sabatini (Addgene plasmid \#85681)

220 (34). Individual sgRNAs were cloned as previously described (35) . In short, sgRNA targeting

221 sequences were annealed and phosphorylated then cloned into a dephosphorylated and BsmBI

222 (New England Biolabs) digested SgOpti. sgRNA constructs were then packaged into lentivirus as

223 previously described and used to transduce early passage FLAMs. Two days later, transductants

224 were selected with puromycin. After one week of selection, gDNA was isolated from each 
225 targeted FLAM, and PCR was used to amplify edited regions and sanger sequencing was used to

226 quantify indels.

227

Construction of genome-wide loss-of-function library and Siglec-F Screen

229 The mouse BRIE knockout CRISPR pooled library was a gift of David Root and John Doench

230 (Addgene \#73633) (36). Using the BRIE library, 4 sgRNAs targeting every coding gene in mice in

231 addition to 1000 non-targeting controls (78,637 sgRNAs total) were packaged into lentivirus

232 using HEK293T cells and transduced Cas9 ${ }^{+}$FLAMs at a low multiplicity of infection (MOI <0.3).

233 Two days later these cells were selected with puromycin. We then passaged the transduced

234 library in TGF $\beta$ in parallel with non-transduced cells of the same passage without TGF $\beta$. When

235 the non-transduced cells grown in the absence of TGF- $\beta$ showed reduced Siglec-F expression

236 by flow cytometry we isolated gDNA from the library for sequencing and found high coverage

237 and distribution (Table S1). In parallel, the transduced library was fixed, and fluorescence

238 activated cell sorting (FACS) was used to isolate the SiglecF ${ }^{\text {high }}$ and SiglecF ${ }^{\text {low }}$ bins using a BioRad

239 S3e cell sorter. Genomic DNA was isolated from each sorted population from two biological

240 replicate experiments using a homemade modified salt precipitation method previously

241 described (37). Amplification of sgRNAs by PCR was performed as previously described using

242 Illumina compatible primers from IDT (36), and amplicons were sequenced on an Illumina

243 NovaSeq 6000 at the RTSF Genomics Core at Michigan State University.

245 Sequence reads were first trimmed to remove any adapter sequence and to adjust for p5

246 primer stagger. We used MAGeCK to map reads to the sgRNA library index without allowing for

247 any mismatch. Subsequent sgRNA counts were median normalized to control sgRNAs in

248 MAGeCK to account for variable sequencing depth. To test for sgRNA and gene enrichment, we

249 used the 'test' command in MAGeCK to compare the distribution of sgRNAs in the SiglecF ${ }^{\text {high }}$

250 and SiglecF ${ }^{\text {low }}$ bins.

251

\section{Bioinformatic analysis}


253 Both DAVID analysis and GSEA analysis were used to identify enriched pathways and protein

254 families that were enriched in the data set. Genes were ranked in MAGeCK using RRA and the

255 top enriched positive regulators (4-fold change with at least 2 sgRNAs) were used as a

256 "candidate list" in both DAVID analysis using default settings (38). Functional analysis and

257 functional annotation analysis were completed, and top enriched pathways and protein families

258 were identified. For GSEA analysis, the "GSEA Preranked" function was used to complete

259 functional enrichment using default settings for KEGG, Reactome and GO terms.

Data availability

262 Raw sequencing data in FASTQ and processed formats will be available for download from NCBI 263 Gene Expression Omnibus (GEO) and available upon request.

\section{Statistical analysis and data visualization}

Statistical analysis and data visualization were performed using Prism Version 8 (GraphPad) or R studio as indicated in the figure legends. SYTOX ${ }^{+}$and $\mathrm{CSiO}_{2}$-filled cells were quantified using CellProfiler. Data are presented, unless otherwise indicated, as the mean \pm the standard deviation. For parametric data, one-way ANOVA followed by Tukey's post-hoc test was used to identify significant differences between multiple groups, and Student's t-tests were used to

271 compare two groups. Non-parametric one-way ANOVAs and Mann-Whitney U tests were used

272 to compare multiple groups and two groups, respectively, for non-parametric data.

\section{RESULTS}

276 Fetal liver-derived cells and AMs cultured in GM-CSF alone do not stably express lineage-

277 specific markers over time

278 The development of a genetically tractable AM ex vivo model requires a stable population that

279 maintains AM-like phenotypes and functions long-term. As a first step, we examined the long-

280 term stability of AM-like cells using a previously described method culturing fetal liver derived

281 cells in the cytokine GM-CSF (25). Consistent with previous reports, we found that fetal liver 
cells grown in GM-CSF phenotypically and morphologically resemble $\operatorname{AMs}(25,39,40)$. Fetal

283 liver cells grown for 2 weeks ex vivo in the presence of GM-CSF adopt a distinct fried-egg-like

284 morphology akin to AMs (Figure 1A). Scanning electron microscopy revealed that the surfaces

285 of both AM and low passage fetal liver cells (<1 month of culture) have numerous outer

286 membrane ruffles (Figure 1B). However, high passage fetal liver cells (>1 month of culture)

287 underwent a morphological shift from an AM-like, ovoid morphology with numerous outer

288 plasma membrane ruffles, to a smaller, fusiform morphology with loss of membrane ruffles

289 (Figure 1A and 1B). Thus, in our hands, the morphology of fetal liver derived cells grown in

290 recombinant GM-CSF are not stable long-term.

292 We further examined whether changes in surface markers or gene expression varied as fetal

293 liver cells were cultured over time. Using flow cytometry, we found similarity between AMs and

294 low passage fetal liver cells with high surface expression of Siglec-F and CD11c and low

295 expression of CD14. However, high passage fetal liver cells showed low expression of SiglecF

296 and CD11C, while expressing high levels of CD14 (Figure 1C). Similarly, when we quantified gene

297 expression, we observed that low passage fetal liver cells and AMs express high levels of Pparg,

298 Car4, II1a and Fabp4 (a transcriptional target of PPAR $\gamma$ ) and low levels of CD14, while high

299 passage fetal liver cells expressed very low levels of the AM-associated transcripts but high

300 levels of CD14 (Figure 1D). We observed similar results with AMs isolated from the lungs

301 (Figure S1A and B). To exclude the possibility of contaminating cells outcompeting the alveolar-

302 like cells over long-term culture we used fluorescence activated cell sorting (FACS) to isolate a

303 pure Siglec-F positive population of cells that were then cultured in GM-CSF media. We

304 continued to observe a decline in Siglec-F and CD11c in these cells (Figure S2). Together these

305 data suggest that prolonged culture of fetal liver-derived cells in GM-CSF media results in a

306 decline in AM-like properties. 
310 We next pursued strategies to improve the stability of AM-specific phenotypes of fetal liver-

311 derived cells grown ex vivo. Based on a prior report that the cytokine TGF $\beta$ is critical to AM

312 development and homeostasis (10), we hypothesized that the addition of TGF $\beta$ to our culture

313 system would maintain cells in an AM-like state. We first tested whether the addition of TFG $\beta$

314 alters the expression of AM-associated genes. Fetal liver cells in GM-CSF media were treated for

31524 hours with $10 \mathrm{ng} / \mathrm{mL}$ TGF $\beta$, which we found induced the genes Pparg, Car4 a transcriptional

316 target of PPAR- - , and Itgax (Figure S3A), all of which are highly expressed by AMs (Figure 1D).

317 We next examined if continued supplementation with TGF $\beta$ stabilizes the AM-like phenotypes

318 of fetal liver cells long-term. Fetal liver-derived cells were cultured in GM-CSF media or GM-CSF

319 media containing $20 \mathrm{ng} / \mathrm{mL}$ TGF $\beta$. After 15 passages (approximately 2 months of culturing), cells

320 grown in the presence of TGF $\beta$ retained a round, AM-like morphology (Figure $2 \mathrm{~A}$ ) and

321 continued expressing AM-identifying genes (Figure 2B). Conversely, fetal liver-derived cells

322 cultured without TGF $\beta$ lost the expression of AM-identifying genes Siglecf, Marco and Pparg

323 and began expressing $C d 14$, which is a common marker for monocyte-derived macrophages

324 recruited to the lung (41) (Figure 2B).

326 We next determined if fetal liver-derived cells grown in the absence of TGF $\beta$ would revert to

327 the AM-like state upon the addition of TGF $\beta$. Fetal liver cells grown in the absence of TGF $\beta$

328 were cultured with and without TGF $\beta$ for 6 days and the expression of Siglec-F and CD14 was

329 quantified by flow cytometry (Figure S3B). In parallel, fetal liver cells maintained in TGF $\beta$ were

330 cultured for six days in the presence or absence of TGF $\beta$. We observed that while the removal

331 of TGF $\beta$ resulted in a significant decrease in Siglec- $F$ and an increase in CD14, there was no

332 change in expression upon the addition of TGF $\beta$ to fetal liver cells that previously lost AM-like

333 marker expression. When we examined the gene expression of Tgfb1 and the TGF $\beta$ receptors,

334 Tgfbr1 and Tgfbr2 we observed a significant decrease in expression of the Tgfb1 (Figure 2B,

335 Figure S3C). These data suggest that the loss of AM-like potential of fetal liver cells grown in the 336 absence of TGF $\beta$ is not reversible. 
338 We next quantified changes in the surface expression of Siglec-F and CD14 by flow cytometry in

339 the fetal liver-derived cells grown in the presence and absence of TGF $\beta$. Consistent with our

340 gene expression analysis we observed that fetal liver-derived cells lose the expression of Siglec-

$341 \mathrm{~F}$ and gain the expression of CD14 over time (Figure 2C and 2D). In contrast, fetal liver-derived

342 cells grown with TGF $\beta$ maintained over $80 \%$ of cells with high levels of Siglec-F expression and

343 low levels of CD14. Thus, culturing fetal-liver cells in both GM-CSF and TGF $\beta$ results in the stable

344 gene expression of self-replicating cells that phenotypically resemble AMs.

346 Fetal liver-derived cells grown in GM-CSF and TGF $\beta$ are functionally similar to AMs in

347 response to $\mathrm{CSiO}_{2}$ relative to phagocytosis, IL-1 cytokine release, and death

348 To assess the functional similarity of fetal liver-derived cells grown in TGF $\beta$ with AMs, we

349 assessed the response of cells to crystalline silica $\left(\mathrm{cSiO}_{2}\right)$, a respirable particle associated with

350 silicosis and autoimmunity $(42,43)$. Cells were exposed to various concentrations of $\mathrm{cSiO}_{2}$ for 8

351 hours. SYTOX Green, a membrane impermeable nucleic acid stain, was included to assess lytic

352 cell death. AMs and low passage fetal liver-derived cells grown with and without TGF $\beta$ showed

353 similar rates of $\mathrm{CSiO}_{2}$ engulfment and cell death (Figure 3A). However, late passage fetal liver-

354 derived cells without TGF $\beta$ exhibited poor phagocytosis, and as a result, tolerated the presence

355 of $\mathrm{CSiO}_{2}$ without inducing cell death. This unresponsiveness is prevented by TGF $\beta$, as late

356 passage fetal liver-derived cells effectively phagocytosed $\mathrm{CSiO}_{2}$ (Figure 3A and 3B). Rates of

357 phagocytosis by BMDMs were comparable to AMs but were accompanied by a two-fold

358 increase in cell death. Thus, fetal liver-derived cells grown in TGF $\beta$ and GM-CSF are functionally

359 stable long-term and recapitulate phagocytosis and cell death kinetics similarly to AMs.

361 IL-1 $\alpha$ is associated with the inflammatory response to particle-induced inflammation. In vivo

362 and ex vivo studies suggest AMs are the primary source of IL-1 $\alpha$ in the lung following inhalation

363 of $\mathrm{CSiO}_{2}$, likely as a result of cell death $(44,45)$. Initial characterizations of fetal liver-derived

364 cells grown in GM-CSF (30) showed they respond to LPS like AMs by producing high levels of IL-

$3651 \alpha$ and low levels of IL-10 in contrast BMDMs that make little IL-1 $\alpha$. We replicated these

366 experiments and observed similar results with low passage fetal liver-derived cells producing 
367 high levels of IL-1 $\alpha$ and low levels of IL-10 in response to LPS (Figure S3D). We next tested how

368 the IL-1 $\alpha$ response to $\mathrm{CSiO}_{2}$ differed over-time in fetal liver-derived cells grown in the presence

369 and absence of TGF $\beta$. We found that high levels of IL-1 $\alpha$ were released both low and high

370 passage fetal liver-derived cells grown in both GM-CSF and TGF $\beta$ following $\mathrm{CSiO}_{2}$ exposure for 8

371 hours, similar to AMs (Figure $3 \mathrm{C}$ ). In contrast, we observed that $\mathrm{CSiO}_{2}$ induced IL-1 $\alpha$ release

372 from low passage fetal liver-derived cells grown in GM-CSF alone but not late passage cells. Late

373 passage cells grown in GM-CSF alone instead phenocopied BMDMs and released no detectable

374 IL-1 $\alpha$ following $\mathrm{CSiO}_{2}$ exposure. Release of IL-1 $\beta$ in these cells may be indicative of

375 inflammasome activation, which is a major mechanism of AM toxicity following exposure to

$376 \mathrm{cSiO}_{2}$. We found $\mathrm{CSiO}_{2}$ exposure to elicit modest IL-1 $\beta$ release from low passage fetal liver-

377 derived cells grown without TGF $\beta$, and from both low and high passage fetal liver-derived cells

378 grown with TGF $\beta$. We further observed a slight, though not significant, increase in IL-1 $\beta$ release

379 in $\mathrm{AMs}$ following $\mathrm{cSiO}_{2}$ exposure (Figure $3 \mathrm{C}$ ). $\mathrm{cSiO}_{2}$-induced IL-1 $\beta$ release was not evident from

380 BMDMs or late MPI cells. Taken together, these experiments show that growth of fetal-liver

381 cells in both GM-CSF and TGF $\beta$ recapitulates many aspects of AM physiology and function as

382 stable, long-term, self-propagating cells. We call these cells Fetal Liver-derived Alveolar

383 Macrophages (FLAMs).

\section{CRISPR-Cas9 editing in FLAMs enables disruption of AM-specific responses to cSiO2. A}

386 significant hinderance in the study of AMs is their intractability to standard genetic approaches.

387 This shortcoming has limited the understanding of pathways and regulators that control AM

388 maintenance and function. We hypothesized that FLAMs could be leveraged to dissect AM

389 functional mechanisms. To test this hypothesis we developed CRISPR-Cas9 mediated gene-

390 editing tools by generating FLAMs from Cas9+ mice $(46,47)$. Using these cells, we targeted

391 Marco and $/ / 1 r 1$, two genes associated with phagocytosis and inflammatory responses to $\mathrm{cSiO}_{2}$

392 in AMs $(48,49)$. Each gene was targeted using two independent sgRNAs per gene by lentiviral

393 transduction. Following selection of successfully transduced cells, we evaluated the editing

394 efficiency of each target genes using Tracking of Indels by DEcomposition (TIDE) analysis. We

395 observed robust editing for both sgRNAs with at least one sgRNA per gene reaching over 95\% 
editing efficiency (Figure S5A,B). Thus, FLAMs are amenable to genetic targeting by CRISPR-

397 Cas9.

Given the scavenger receptor MARCO has been shown to be involved in $\mathrm{CSiO}_{2}$ uptake and toxicity while IL1R1 is known to amplify inflammatory cues, we hypothesized that cells deficient

401 in MARCO and IL1R1 expression would have a reduced inflammatory response to $\mathrm{CSiO}_{2}(48,50)$.

402 We therefore tested whether FLAMs targeted for Marco or I/1r1 would differentially respond to $403 \mathrm{CSiO}_{2}$ exposure compared to wild-type. We exposed control FLAMs and sgMarco or sgll1r1 404 FLAMs to two different $\mathrm{CSiO}_{2}$ concentrations and quantified cell death. While we observed no 405 change in cell death in sgll1r1 FLAMs compared to control FLAMs, a significant reduction in cell 406 death in sgMarco FLAMs was observed following high $\mathrm{cSiO}_{2}$ exposure (Figure 4A). We next 407 examined the production of IL1 following exposure of cells to $\mathrm{cSiO}_{2}$. We found reduced $\mathrm{cSiO}_{2-}$ 408 induced IL-1 $\alpha$ and IL-1 $\beta$ production by sgMarco and sgll1r1 FLAMs compared to control FLAMs 409 (Figure 4B,C). Therefore, FLAMs are genetically tractable and can be used to dissect AM-specific 410 functions.

Forward genetic screen in FLAMs identifies regulators of the AM surface marker Siglec-F.

413 The genetic tractability of FLAMs opens the possibility of performing forward genetic screens in 414 an AM context, which was previously unviable. We recently developed a screening platform in 415 immortalized bone marrow macrophages (iBMDMs) that uses cell sorting of CRISPR-Cas9 416 targeted cells to enrich for genes that positively or negatively regulate the surface expression of 417 important immune molecules (51). We hypothesized this screening pipeline could be leveraged 418 to dissect pathways responsible for the unique expression profiles seen in AMs and FLAMs. As a 419 first step to test this hypothesis, we dissected the changes in the surface expression of Siglec- $F$ 420 when targeted using CRISPR-Cas9. Among macrophages, Siglec-F is uniquely expressed on the 421 surface of AMs, yet how Siglec-F is regulated remains entirely unknown. Given that Siglec- $F$ 422 expression is lost as cells lose their AM-like phenotypes, globally understanding Siglec-F 423 regulation in FLAMs may inform key gene networks in AMs. To test the dynamic range of Siglec424 F expression on FLAMs, we targeted Siglec-F with two independent sgRNAs in both Cas9 ${ }^{+}$ 
425 FLAMs and iBMDMs. Again, extensive editing for both sgRNAs was observed with one sgRNA

426 reaching over 99\% editing efficiency (Figure S5C). As expected, control iBMDMs showed no

427 surface Siglec-F expression and targeting Siglec-F showed no observable change by flow

428 cytometry (Figure 5A). In contrast, we observed robust Siglec-F expression on control FLAMs

429 while sgSiglecF FLAMs showed a greater than 100-fold reduction in MFI (Figure 5B). This

430 dynamic range is comparable to other surface markers we previously screened in iBMDMs,

431 suggesting that Siglec-F is an ideal target for a genetic screen in FLAMs (51).

433 To globally identify genes that contribute to Siglec-F surface expression on FLAMs, we

434 generated a genome-wide knockout library. FLAMs from Cas9 ${ }^{+}$mice were transduced with

435 sgRNAs from the pooled Brie library (52) which contains 4 independent sgRNAs per mouse

436 coding gene. In parallel to the library, we grew control Cas $9^{+}$fetal liver cells with GM-CSF alone

437 to monitor the loss of Siglec-F expression in the absence of TGF $\beta$ signaling (Figure 5C and 5D).

438 When control cells lost Siglec-F expression, genomic DNA from the FLAMs knockout library was

439 purified and the sgRNAs were quantified by deep sequencing. The library coverage was

440 confirmed to have minimal skew (Table S1). We then conducted a forward genetic screen using

441 FACS to isolate the Siglec- $\mathrm{F}^{\text {high }}$ and Siglec- $\mathrm{F}^{\text {low }}$ cells from the loss-of-function FLAM library (Figure

442 5C). Following genomic DNA extraction, sgRNA abundances for each sorted population were

443 determined by deep sequencing. To test for statistical enrichment of sgRNAs and genes, we

444 used the modified robust rank algorithm ( $\alpha$-RRA) employed by Model-based Analysis of

445 Genome-wide CRISPR/Cas9 Knockout (MAGeCK). MAGeCK first ranks sgRNAs by effect and then

446 filters low ranking sgRNAs to improve gene significance testing (53). To identify genes that are

447 required for Siglec-F expression we compared the enrichment of sgRNAs in the Siglec- $F^{\text {low }}$

448 population to the Siglec- $F^{\text {high }}$ population. The $\alpha-$ RRA analysis identified over 300 genes with a $p$ -

449 value $<0.01$ and the second ranked gene in this analysis was the target of the screen Siglec- $F$

450 (Figure 5E and Table S2). Guide-level analysis showed agreement with all four sgRNAs targeting

451 Siglec- $F$, with each showing a ten-fold enrichment in the Siglec- $F^{\text {low }}$ population (Figure 5F). The

452 high ranking of Siglec-F gives high confidence in genome-wide screen results. 
454 Stringent analysis revealed an enrichment of genes with no previously described role in Siglec- $F$

455 regulation including the TGF $\beta$ response regulator USP9x (54) (Figure 6A). To identify pathways

456 that were associated among these genes, we filtered the ranked list to include genes that had a

457 fold change of $>4$ with at least 2 out of 4 sgRNAs and used DAVID analysis to identify pathways

458 and functions that were enriched in our datasets. The top enriched KEGG pathway was the

459 peroxisome, with all core components of peroxisome biogenesis identified as positive

460 regulators of Siglec-F (Figure 6B). We further examined other peroxisome-associated (PEX)

461 genes and found that 11 out of 15 PEX genes present in our library were altered greater than

462 two-fold (Figure 6C). KEGG pathway analysis identified a significant enrichment in genes

463 associated with lipid metabolism, including glycerophospholipid, inositol phosphate, and ether

464 lipids (Table S3). KEGG analysis also found an enrichment of the phagosome pathway which

465 identified several surface receptors associated with phagocytosis in this pathway, including the

466 IgG Fc Receptor 4, the mannose-6-phosphate receptor, and the oxidized low-density lipoprotein

467 receptor, suggesting surface proteins associated with phagocytosis directly modulate the

468 stability of Siglec-F (Figure 6D). Examination of enriched UniProt keyword terms using DAVID

469 analysis found a strong enrichment of proteins with oxidoreductase function including several

470 genes associated with cytochromes P450 (CYP), a key regulator of xenobiotic, fatty acid, and

471 hormone metabolism, known to be important in the lung environment (55). Thus, bioinformatic

472 analysis of the top positive regulators of Siglec-F identified pathways that are associated with

473 AM functions.

475 We next used gene set enrichment analysis (GSEA) to identify functional enrichments from the 476 entire ranked screen dataset (Table S3). GSEA identified the peroxisome as a top enriched

477 KEGG pathway consistent with the DAVID analysis (Figure 6E). This analysis also identified a

478 strong enrichment for oxidative phosphorylation, which is consistent with the key metabolic

479 changes in AMs compared to BMDMs (56), and a significant enrichment for GPI anchor

480 synthesis as negative regulators of Siglec-F surface expression (Figure 6E). We also noted that

481 mTORC1 signaling was enriched as a negative regulator, in line with previous reports that

482 mTORC1 is required to maintain AMs in the lungs (57). Taken together, our forward genetic 
483 screen not only identified Siglec- $F$, the screen target, but also identified positive and negative

484 regulators of Siglec-F expression that are associated with known AM-functions as well as novel

485 AM regulators. Thus, FLAMs are a tractable genetic platform that enables the detailed

486 interrogation of AM regulatory functions and mechanisms.

\section{DISCUSSION}

As long-lived resident macrophages in the lungs, AMs have unique phenotypes and functions

491 shaped by the alveolar environment (58). However, experimental limitations hinder our

492 understanding of AM-specific functional mechanisms. Developing ex vivo models that

493 recapitulate AM phenotypes would overcome the challenges associated with isolating and

494 maintaining AMs from the lungs of mice. Since AMs are derived from fetal liver monocytes,

495 previous studies tested the culture of fetal liver cells with GM-CSF (59). These culture

496 conditions result in self-replicating AM-like cells, but in our hands, the AM-like phenotype was

497 not stable long-term. While low passage fetal liver cells grown in GM-CSF are useful for some

498 experimental approaches, the instability of the AM phenotype precludes functional genetic

499 studies $(60,61)$. To stabilize the AM-like phenotype of fetal liver-derived cells we supplemented

500 the growth media with TGF $\beta$, a key cytokine for AM maintenance in the lungs, in a model we

501 term FLAMs (10). Here we showed that FLAMs recapitulate many aspects of AM biology, are

502 stable long-term, and are genetically tractable, making them a useful tool to dissect the

503 regulation of $A M$ maintenance and function.

504

505 We demonstrated that that even after one month of culture ' FLAMs efficiently phagocytose

$506 \mathrm{CSiO}_{2}$ particles, produce inflammatory cytokines like IL-1 $\alpha$, and die similarly to AMs. Our results

507 are consistent with two recent reports that examined how TGF $\beta$ modulates macrophages ex

508 vivo $(60,61)$. These reports showed that TGF $\beta$ can induce/maintain AM-like phenotypes ex vivo

509 using AMs directly from the lungs of mice or purified cells from the bone-marrow. Other

510 differences in these approaches, including the use of the PPAR $\gamma$ agonist rosiglitazone, make

511 these strategies distinct yet complementary and underscore the key role of TGF $\beta$ in AM 
512 regulation. The advantage of FLAMs is the low cost, low technology threshold and high yield of

513 cells that can be isolated from any genetically modified mouse. Another key advantage of

514 FLAMs is the potential of the genetic tools developed here. Using targeted gene-editing we

515 showed that directed mutations can be easily generated in FLAMs to probe specific AM

516 functions. Furthermore, we generated a genome-wide knockout library in FLAMs and

517 completed the first forward genetic screen in AM-like cells, highlighting the utility of FLAMs as a

518 genetic platform to probe AM function. Thus, FLAMs recapitulate ex vivo AMs even after

519 extended culturing and are suitable for dissecting AM responses and regulation.

521 How AMs control their functional responses and how this differs from other macrophage

522 populations remains unclear. Here we observed both phenotypic and functional differences

523 among AMs, FLAMs, and BMDMs in line with previous studies $(25,39,40)$. While BMDMs

524 express high levels of CD14, AMs and FLAMs express high levels of Siglec-F and MARCO. When

525 cells were exposed to $\mathrm{CSiO}_{2}$, we observed differences in cell death kinetics and IL-1 cytokine

526 responses. Though $\mathrm{BMDMs}$ were able to engulf $\mathrm{cSiO}_{2}$ particles at a rate comparable to $\mathrm{AMs}$ and

527 FLAMs, they quickly succumbed to cell death, while AMs and FLAMs remained viable many

528 hours following $\mathrm{CSiO}_{2}$ phagocytosis. A delay in cell death may be important for appropriate

529 clearance of particles, potentially allowing the AMs to be transported out of the alveoli before

530 they die (62). AMs and AM-like cells also released significantly more IL-1 $\alpha$ and IL-1 $\beta$ than

531 BMDMs in response to $\mathrm{CSiO}_{2}$. These data are consistent with studies showing high levels of IL-1 $\alpha$

532 produced by AMs compared to other cells in the lung and other macrophage sub-types $(25,39$,

$53344,45,59)$ and the known role of $\mathrm{CSiO}_{2}$ in inducing IL-1 $\beta$ release (49). Our findings indicate that

534 MARCO may be a key player in driving the IL-1 cytokine response in AMs as MARCO-deficient

535 FLAMs showed increased viability and decreased IL-1 production following $\mathrm{CSiO}_{2}$ exposure.

536 These results are in line with previous studies implicating MARCO in the uptake of $\mathrm{cSiO}_{2}$ and

537 other particles in $\operatorname{AMs}(50,63)$. In the future, FLAMs will be used to dissect the underlying

538 mechanisms of MARCO regulation to understand how MARCO drives distinct inflammatory

539 responses following phagocytosis of $\mathrm{cSiO}_{2}$ and other pathogenic cargo. Knocking out the IL-1 
540 receptor also reduced IL-1 cytokine release, which points to a feed-forward mechanism to

541 amplify this inflammatory response in AMs.

542

543 In addition to MARCO, AMs express other markers that are used to define AM populations.

544 However, the regulation of these other AM markers, like Siglec- $F$, remains entirely unknown.

545 Siglec- $\mathrm{F}$ is a surface-expressed immunoglobulin protein that binds sialic acid residues on

546 glycolipids and glycoproteins, but its function in AMs is largely unknown. In addition to AMs,

547 Siglec- $F$ is expressed on eosinophils, where it limits inflammation by modulating cell death

548 pathways (64). The only studies examining Siglec-F in AMs demonstrated that Siglec-F does not

549 regulate phagocytic activity (65). Our forward genetic screen in FLAMs defined regulators of

550 Siglec-F surface expression and uncovered hundreds of candidate genes that may contribute to

551 Siglec-F expression. Our results not only identified Siglec-F as the second ranked candidate, but

552 we identified other genes that likely modulate Siglec-F expression or trafficking. These genes

553 include transcription factors like Fos and NFkB2 and surface receptors like M6PR. Our screen

554 candidates are likely to include both direct regulators of Siglec-F expression and indirect

555 regulators that maintain the AM-like state. In support of this prediction, we identified USP9x, a

556 known regulator of TGF $\beta$ signaling as a strong positive regulator of Siglec-F expression (54). In

557 addition, we identified the enrichment of functional pathways previously associated with AM

558 function including the peroxisome, lipid metabolism, oxidative phosphorylation and CYP. Given

559 the previous links among PPAR transcription factors, peroxisome biogenesis, and lipid

560 metabolism, our data strongly suggest FLAMs recapitulate the metabolic makeup of AMs which

561 is central to their gene regulation $(12,56,66)$. In further relation to the metabolic state of

562 FLAMs, we identified several CYP family members among our top candidates which regulate

563 vitamin A and all-trans retinoic acid, known modulators of AM function $(67,68)$. Based on these

564 findings, we posit that PPAR

565 transcriptional profile, resulting in Siglec-F expression. Future studies will be centered on

566 testing this model and deeply validating the genetic screen to uncover novel regulatory

567 mechanisms in FLAMs. 
569 FLAMs are a promising model to study AM biology, yet some limitations remain. While FLAMs

570 maintain many AM-like phenotypes long-term, we observed variable expression of the AM

571 marker CD11c over time. This suggests that there are other signals in addition to GM-CSF and

572 TGF $\beta$ that are needed to fully recapitulate AM functionality ex vivo. The alveolar space is a

573 highly complex microenvironment, with constant crosstalk between AMs and other cells (58).

574 For example, our data show that FLAMs express TGF $\beta$, yet this is not sufficient to maintain AM-

575 like functions and continued Tgfbr1 and Tgfbr2 expression. Given TGF $\beta$ is known to amplify

576 Tgfbr1 and Tgfbr2 this suggests that the TGF $\beta$ produced by FLAMs is not biologically active. In

577 vivo, latent TGF $\beta$ released by AMs is activated by $\alpha-V-\beta-6$ integrins expressed on the type II

578 alveolar epithelial cells (AECII), resulting in increased levels of the active protein which can

579 signal in an autocrine manner to maintain the unique phenotype of AMs (69). This feed-forward

580 loop is absent ex vivo, which may explain why addition of exogenous active TGF $\beta$ prevents the

581 loss of the AM-like phenotype in FLAMs. Other signals provided by AECII cells and others such

582 as lung-resident basophils likely regulate AM maintenance, but these signals are not modeled in

583 our system (70). Combining the genetic tractability of FLAMs with in vivo transfer models may

584 enable us in the future to dissect this cross-regulation systematically to further improve FLAMs.

585 Intranasal transfer of TGF $\beta$-cultured AMs was recently shown to repopulate the alveolar space

586 suggesting FLAMs could be similarly instilled, enabling rapid studies to better understand AM-

587 maintenance within the lung environment (27).

589 In summary, we developed FLAMs, a stable ex vivo model that can be used to study lung 590 development, immunology, and toxicology. FLAMs are likely to shed new light on processes

591 unique to AMs, like phagocytosis, efferocytosis and the removal of inhaled particles, by

592 employing targeted or genome-wide genetic approaches. Taken together, the optimization and

593 application of FLAMs provides an exciting, innovative model to thoroughly investigate AM

594 biology.

595

596 Acknowledgements. 
598 We would like to acknowledge Dr. Jack Harkema, Dr. Melissa Bates, Dr. Mikhail Givralin, Dr.

599 Alexander Misharin, and the members of the Olive and Pestka lab for helpful discussions and

600 input. We thank the MSU flow cytometry core for their help with instrumentation and analysis

601 and Carol Flegler of the MSU Center of Advanced Microscopy for assistance in scanning

602 electron microscopy.

603

604 Funding. This work was supported by startup funding to AJO provided by Michigan State

605 University, the Rackham Endowment Award [AJO], the Dr. Robert and Carol Deibel Family

606 Endowment [JP] as well as grants from the NIH(Al148961 [AJO], F31ES030593 [KW],

607 ES027353 [JP] and T32ES030593 [KW]), DOD (W81XWH2010147 [AJO]) and USDA (NIFA

608 HATCH 1019371 [AJO]).

609

610

611 Competing Interests

612 The authors have no competing interests related to the research described in this manuscript.

Figure Legends

617 Figure 1. Fetal liver macrophages cultured with GM-CSF lose their AM-like phenotype over

618 time. Fetal liver cells were cultured with GM-CSF and analyzed at indicated passage. AMs were

619 isolated and analyzed immediately. A) AMs, P2 fetal liver cells, and P37 fetal liver cells were

620 lifted from culture and imaged on a EVOS FL Auto 2 fluorescent microscope at 60x

621 magnification. B) AMs, P3 fetal liver cells, and P15 fetal liver cells were fixed and imaged by

622 scanning electron microscopy at 4500x, 4000x, and 2200x, respectively. C) Alveolar

623 macrophages (Gray), P4 fetal liver macrophages (Blue), and P15 fetal liver macrophages (Red)

624 were assessed for surface expression of the markers CD14, Siglec-F, and CD11c by flow

625 cytometry. D) Gene expression of indicated genes in AMs, early fetal liver macrophages (P1),

626 and late fetal liver macrophages (P18) was quantified by qPCR. Data was compared using one- 
627 way ANOVA followed by Tukey's multiple comparisons test. Bars labeled with unique letters are

628 significantly different $(p<0.05)$. Results are representative of 2 or 3 independent experiments.

Figure 2. Culturing fetal liver cells with TGF $\beta$ and GM-CSF maintains AM-like phenotypes long-

term. Fetal liver cells were cultured with or without TGF $\beta$ for the indicated passages. A) P15

632 cells cultured with and without TGF $\beta$ were imaged on a EVOS FL Auto 2 fluorescent microscope

633 at 60x magnification. Cells with a clearly visible spindleoid morphology are marked with arrows.

634 B) At the indicated passage, RNA was extracted from a subset of cells for gene expression

635 analysis. Expression of the indicated genes are quantified as relative copy number (RCN)

636 compared to Gapdh. Asterisks indicate significant $(p<0.05)$ differences in gene expression of

637 cells cultured with and without TGF- $\beta$ cells at the same passage number, as determined by

638 Student's t-test. C) At the indicated passage, cells were analyzed by flow cytometry for the

639 surface expression of CD14 and Siglec-F. Representative biaxial plots from triplicate samples is

640 shown. D) Quantification of the mean fluorescence intensity (MFI) and percent of cells positive

641 of CD14 and Siglec-F surface expression from cells in (C) expressed as MFI (left) and percent

642 positive (right). Results are representative of at least two independent experiments.

$643 * * * * p<.0001$ by one-way ANOVA with a Tukey correction for multiple comparisons.

Figure 3. The kinetics of $\mathrm{CSiO}_{2}$ uptake and $\mathrm{cSiO}_{2}$-induced cell death and IL-1 release are similar

646 among AMs and FLAMs. AMs, bone marrow-derived macrophages (BMDMs), and fetal liver

647 cells were seeded in 96-well plates. After 24 hours, media was replaced with FluoroBrite DMEM

648 containing $200 \mathrm{nM}$ SYTOX green and 10\% FBS. $\mathrm{CSiO}_{2}$ at the indicated densities was added

649 dropwise to cells and images were taken at 0, 2, 6, and 8 hours using an EVOS FL2 fluorescent

650 microscope. A) The percent of $\mathrm{CSiO}_{2}$-filled and SYTOX ${ }^{+}$cells were quantified using the

651 CellProfiler software. B) Representative images of $\mathrm{SYTOX}^{+}$and $\mathrm{cSiO}_{2}$-filled cells (white arrows in

652 AM panel, top right) treated with $50 \mu \mathrm{g} / \mathrm{cm}^{2}$ silica for 8 hours, 20x magnification. C) In a

653 separate experiment, the supernatant was collected after $8 \mathrm{hr}$ treatment with $25 \mu \mathrm{g} / \mathrm{cm}^{2}$ to

654 assess release of the cytokines IL-1 $\alpha$ (Top) and IL-1 (Bottom) by ELISA. Asterisks indicative of

655 significant differences between groups ( $\left.{ }^{* *} p<0.01, * * * p<0.001\right)$, as assessed by Student's t-tests 
656 between relevant groups. ND = not detected. Results are representative of at least two

657 independent experiments.

Figure 4. The loss of Marco and $/ 11 \mathrm{r}$ modulate the response of FLAMs to $\mathrm{CSiO}_{2}$ treatment. Wild

type, Marco KO, and I/1r1 KO FLAMs were treated with $\mathrm{CSiO}_{2}$ at the indicated concentrations for 8 hours. A) Cell viability was determined using the MTS assay, with $100 \%$ viability determined as the mean absorbance of the formazan dye product in the untreated WT cells. Supernatant was collected to measure release of B) IL-1 $\alpha$ and C) IL-1 $\beta$. Asterisks indicate significant differences

$664\left({ }^{*} p<0.05, * * * p<0.001\right)$ between cell types within treatment groups, as determined by one-way ANOVA. Results are representative of at least two independent experiments with biological triplicates. Figure 5. A loss-of-function forward genetic screen identifies regulators of Siglec-F surface expression on FLAMs. iBMDMs or FLAMs targeted for IIr1 or SiglecF were analyzed by flow cytometry for Siglec-F surface expression. A) Shown are representative histograms of surface

671 expression. B) The MFI of Siglec-F surface expression was quantified on cells of the indicated 672 genotypes. $* * * * p<0.0001$ between samples by one-way ANOVA with a Tukey correction. These 673 data are representative of two independent experiments C) Shown is a schematic of the 674 generation of the FLAM knockout library and screen to identify Siglec-F regulators. The 675 transduction of Cas9+ FLAMs with genome-wide library of sgRNAs results in variable Siglec-F surface expression. When parallel control FLAMs grown in the absence of TGF $\beta$ lost Siglec- $F$

677 expression the top and bottom 5\% of Siglec-F expression cells were isolated from the knockout 678 FLAM library by FACS. Sorted cells were then used for downstream sequencing and analysis. D) 679 Siglec-F surface expression of library control cells grown in the absence of TGF $\beta$ was monitored 680 over time and compared to the transduced FLAM library prior to sorting. Shown is the MFI for 681 Siglec-F expression of the indicated cells and passage numbers E) Shown is the $\alpha$-RRA score of 682 each gene in CRISPR library that passed filtering metrics in MAGeCK. Genes of interest are 683 noted. F) Normalized sgSiglecF counts for each sgRNA found in both the SiglecF ${ }^{\text {low }}$ and 684 SiglecF ${ }^{\text {high }}$ sorted populations is shown. 
Figure 6. Bioinformatic analysis identifies FLAM metabolic networks as critical regulators of

687 Siglec-F expression. A) The TGF $\beta$ response regulator USP9x was a significant hit in the screen.

688 Shown are the normalized counts for each of the four sgRNAs targeting USP9x in each sorted

689 population. B) Using DAVID analysis, peroxisome biogenesis was identified as the most

690 significantly enriched KEGG pathway. Shown is an adaptation of the KEGG peroxisome

691 biogenesis pathway highlighting the 10 peroxisome regulators identified in the screen in red. C)

692 The sgRNA distribution and mean log-fold change for each peroxisome regulator identified in

693 the genetic screen are shown. The dashed line indicates a $\log _{2}$ fold change of -1 . D) DAVID

694 analysis identified surface proteins associated with phagocytosis. Shown are the normalized

695 counts for each of the four sgRNAs targeting the indicated surface protein from each sorted

696 population. E) GSEA was used to identify enriched pathways from the entire forward genetic

697 screen. Shown are four leading-edge analysis plots that are representative of this analysis for a

698 subset of enriched pathways. These pathways including the peroxisome, oxidative

699 phosphorylation, GPI anchor biosynthesis and mTORC1 signaling.

Figure S1. Fetal liver macrophages sorted to enrich for Siglec-F-expressing cells lose the AM-

702 like expression profile. Fetal liver macrophages were cultured for 1 week to allow for

703 development and expression of Siglec-F and CD11c and were then stained and sorted based on

704 Siglec-F expression with over $98 \%$ of cells expressing high levels of Siglec-F. Cells were then

705 plated in media containing GM-CSF alone and at the indicated passage cells were lifted and

706 stained for Siglec-F and CD11c Asterisks indicated significant differences between samples

$707(* * * p<0.001)$ as determined by a student's T-test. Results are representative of two

708 independent experiments.

710 Figure S2. Long term ex vivo culture of AMs leads to loss of AM-defining genes and surface

711 markers. Alveolar macrophages (AM) were isolated and cultured in RPMI with $20 \mathrm{ng} / \mathrm{mL} \mathrm{GM-}$

712 CSF for approximately 2 months (12 passages). Gene expression (A) and cell surface markers (B) 
713 were compared between cultured AMs and freshly isolated AMs. Asterisks indicate significant

714 differences $(* * * * p<0.0001, * * p<0.01)$ between samples.

715

716 Figure S3. TGF $\beta$ induces and maintains AM-like state in early-passage but not older fetal liver

717 macrophages. A) Freshly isolated fetal liver cells were incubated with $10 \mathrm{ng} / \mathrm{mL}$ TGF $\beta$ for 24

718 hours prior collecting RNA for qPCR analysis of Car4, Itgax and Pparg gene expression. B) Fetal

719 liver macrophages that were low passage $(<4)$ and kept in TGF $\beta$ and those that were high-

720 passage $(>15)$ and cultured without TGF $\beta$ were cultured for 6 days with and without TGF $\beta$. On

721 day 6, cells were lifted and surface expression of Siglec-F and CD14 was assessed by flow

722 cytometry. C) Expression of Tgfbr2 in fetal liver macrophages cultured with and without TGF- $\beta$

723 was assessed at P5 and P7. Asterisks indicate significant differences $\left({ }^{* * * *} p<0.0001\right.$,

$724 * * * p<0.001, * p<0.05)$ between samples, as determined by Student's t-test. (D) Early fetal liver-

725 derived cells and bone marrow-derived macrophages (BMDMs) were treated with $100 \mathrm{ng} / \mathrm{mL}$

726 LPS for 24 hours and cell-free supernatant was analyzed for release of IL-1 $\alpha$ and IL-10 by ELISA.

727 Asterisks indicate significant differences $\left({ }^{* *} p<0.01\right)$ between fetal liver macrophages cells and

728 BMDMs. ND=not detected.

729

730 Figure S5. Quantification of editing efficiency in FLAMs targeted with CRISPR-Cas9. Cas9 ${ }^{+}$

731 FLAMs were transduced with lentivirus carrying sgRNAs targeting the indicated gene. Following

732 selection, genomic DNA was isolated from control and targeted cells and PCR was used to

733 amplify the region encoding each targeted gene. TIDE analysis was used to quantify the editing

734 efficiency of the indels in each cell line using trace plots following Sanger sequencing. Shown is

735 the TIDE analysis profile indicating the percent editing efficiency for each sgRNA targeting A)

736 I/1r1 B) Marco and C) Siglecf. Red bars indicate the presence of indels of the indicated size.

737 Knockout cells used the remainder of the manuscript in Figures 5 and 6 are underlined.

739 Table S1. Quantification of the coverage in the genome-wide knockout library. (Sheet1)

740 Statistics for the CRISPR screens and zero count sgRNAs are quantified. (Sheet2) Normalized 
741 counts for each sgRNA from two biological replicates of the genome-wide knockout library are

742 listed.

743

744 Table S2. Gene and sgRNA analysis for the Siglec-F genome-wide screen. (Sheet1) The gene

745 summary output from MAGeCK RRA analysis is shown based on SiglecF $^{\text {high }} /$ SiglecF $^{\text {low }}$. (Sheet2)

746 The sgRNA summary output from MAGeCK RRA analysis is shown for each sgRNA in the library.

748 Table S3. Bioinformatic analysis of Siglec-F genome-wide screen. Each labeled sheet indicates

749 the type of analysis (DAVID or GSEA) and the lists the pathways or genes that are associated

750 with each analysis.

751

752

753

754

755

1. Davies LC, Jenkins SJ, Allen JE, Taylor PR. 2013. Tissue-resident macrophages. Nat Immunol2013/09/21. 14:986-995.

757

758

759

760

2. Mass E, Ballesteros I, Farlik M, Halbritter F, Gunther P, Crozet L, Jacome-Galarza CE, Handler K, Klughammer J, Kobayashi Y, Gomez-Perdiguero E, Schultze JL, Beyer M, Bock C, Geissmann F. 2016. Specification of tissue-resident macrophages during

\section{organogenesis. Science2016/08/06. 353.}

3. Lavin Y, Winter D, Blecher-Gonen R, David E, Keren-Shaul H, Merad M, Jung S, Amit I. 2014. Tissue-resident macrophage enhancer landscapes are shaped by the local microenvironment. Cell2014/12/07. 159:1312-1326.

4. Hetzel M, Ackermann M, Lachmann N. 2021. Beyond "Big Eaters": The versatile role of alveolar macrophages in health and disease. Int J Mol Sci2021/04/04. 22.

5. Reddy RC, Keshamouni VG, Jaigirdar SH, Zeng X, Leff T, Thannickal VJ, Standiford TJ. 2004. Deactivation of murine alveolar macrophages by peroxisome proliferator-activated receptor-gamma ligands. American journal of physiology Lung cellular and molecular physiology 286.

6. Misharin A v, Morales-Nebreda L, Mutlu GM, Budinger GR, Perlman H. 2013. Flow cytometric analysis of macrophages and dendritic cell subsets in the mouse lung. Am J Respir Cell Mol Biol2013/05/16. 49:503-510.

7. Guilliams M, de Kleer I, Henri S, Post S, Vanhoutte L, de Prijck S, Deswarte K, Malissen B, Hammad H, Lambrecht BN. 2013. Alveolar macrophages develop from fetal monocytes that differentiate into long-lived cells in the first week of life via GM-CSF. J Exp Med2013/09/18. 210:1977-1992.

8. Kopf M, Schneider C, Nobs SP. 2015. The development and function of lung-resident macrophages and dendritic cells. Nat Immunol2014/12/19. 16:36-44. 
9. Ginhoux F, Guilliams M. 2016. Tissue-resident macrophage ontogeny and homeostasis. Immunity2016/03/18. 44:439-449.

10. Yu X, Buttgereit A, Lelios I, Utz SG, Cansever D, Becher B, Greter M. 2017. The cytokine TGF-beta promotes the development and homeostasis of alveolar macrophages. Immunity2017/11/12. 47:903-912 e4.

11. Schneider C, Nobs SP, Kurrer M, Rehrauer H, Thiele C, Kopf M. 2014. Induction of the nuclear receptor PPAR-gamma by the cytokine GM-CSF is critical for the differentiation of fetal monocytes into alveolar macrophages. Nat Immunol2014/09/30. 15:1026-1037.

12. Reddy RC. 2008. Immunomodulatory role of PPAR-gamma in alveolar macrophages. J Investig Med2008/03/05. 56:522-527.

13. Bonfield TL, Farver CF, Barna BP, Malur A, Abraham S, Raychaudhuri B, Kavuru MS, Thomassen MJ. 2003. Peroxisome proliferator-activated receptor-gamma is deficient in alveolar macrophages from patients with alveolar proteinosis. Am J Respir Cell Mol Biol2003/06/14. 29:677-682.

14. Trapnell BC, Nakata K, Bonella F, Campo I, Griese M, Hamilton J, Wang T, Morgan C, Cottin V, McCarthy C. 2019. Pulmonary alveolar proteinosis. Nature Reviews Disease Primers 2019 5:1 5:1-17.

15. Berenson C, Kruzel R, Eberhardt E, Sethi S. 2013. Phagocytic dysfunction of human alveolar macrophages and severity of chronic obstructive pulmonary disease. The Journal of infectious diseases 208:2036-2045.

16. Berenson CS, Kruzel RL, Eberhardt E, Dolnick R, Minderman H, Wallace PK, Sethi S. 2014. Impaired innate immune alveolar macrophage response and the predilection for COPD exacerbations. Thorax https://doi.org/10.1136/thoraxjnl-2013-203669.

17. Liang Z, Zhang Q, Thomas CM, Chana KK, Gibeon D, Barnes PJ, Chung KF, Bhavsar PK, Donnelly LE. 2014. Impaired macrophage phagocytosis of bacteria in severe asthma. Respiratory Research 2014 15:1 15:1-11.

18. Lévêque $M$, le Trionnaire $S$, del Porto $P$, Martin-Chouly $C$. 2017. The impact of impaired macrophage functions in cystic fibrosis disease progression. Journal of Cystic Fibrosis 16:443-453.

19. McCubbrey A, Curtis J. 2013. Efferocytosis and lung disease. Chest 143:1750-1757.

20. Busch CJ, Favret J, Geirsdottir L, Molawi K, Sieweke MH. 2019. Isolation and long-term cultivation of mouse alveolar macrophages. Bio Protoc2020/01/08. 9.

21. Chen YW, Huang MZ, Chen CL, Kuo CY, Yang CY, Chiang-Ni C, Chen YM, Hsieh CM, Wu HY, Kuo ML, Chiu CH, Lai CH. 2020. PM2.5 impairs macrophage functions to exacerbate

22. Dostert C, Petrilli V, van Bruggen R, Steele C, Mossman BT, Tschopp J. 2008. Innate immune activation through Nalp3 inflammasome sensing of asbestos and silica. Science2008/04/12. 320:674-677.

23. Hornung V, Bauernfeind F, Halle A, Samstad EO, Kono H, Rock KL, Fitzgerald KA, Latz E. 2008. Silica crystals and aluminum salts activate the NALP3 inflammasome through phagosomal destabilization. Nature Immunology 9:847-856.

24. Brenner TA, Rice TA, Anderson ED, Percopo CM, Rosenberg HF. 2016. Immortalized MH-S cells lack defining features of primary alveolar macrophages and do not support mouse pneumovirus replication. Immunol Lett2016/02/27. 172:106-112. 
822 25. Fejer G, Wegner MD, Gyory I, Cohen I, Engelhard P, Voronov E, Manke T, Ruzsics Z, Dolken L, Prazeres da Costa O, Branzk N, Huber M, Prasse A, Schneider R, Apte RN, Galanos C, Freudenberg MA. 2013. Nontransformed, GM-CSF-dependent macrophage lines are a unique model to study tissue macrophage functions. Proc Natl Acad Sci U S A2013/05/28. 110:E2191-8.

26. Wierenga KA, Wee J, Gilley KN, Rajasinghe LD, Bates MA, Gavrilin MA, Holian A, Pestka JJ. 2019. Docosahexaenoic acid suppresses silica-induced inflammasome activation and IL-1 cytokine release by interfering with priming signal. Frontiers in Immunology 10.

27. Gorki A, Symmank D, Zahalka S, Lakovits K, Hladik A, Langer B, Maurer B, SexI V, Kain R, Knapp S. 2021. Murine ex vivo Cultured Alveolar Macrophages Provide a Novel Tool to Study Tissue-Resident Macrophage Behavior and Function. American Journal of Respiratory Cell and Molecular Biology https://doi.org/10.1165/RCMB.2021-01900C.

28. Bates MA, Brandenberger C, Langohr I, Kumagai K, Harkema JR, Holian A, Pestka JJ. 2015. Silica triggers inflammation and ectopic lymphoid neogenesis in the lungs in parallel with accelerated onset of systemic autoimmunity and glomerulonephritis in the lupus-prone NZBWF1 mouse. PLoS ONE 10.

29. Bates MA, Akbari P, Gilley KN, Wagner JG, Li N, Kopec AK, Wierenga KA, Jackson-Humbles D, Brandenberger C, Holian A, Benninghoff AD, Harkema JR, Pestka JJ. 2018. Dietary docosahexaenoic acid prevents silica-induced development of pulmonary ectopic germinal centers and glomerulonephritis in the lupus-prone NZBWF1 mouse. Front Immunol2018/09/28. 9:2002.

30. Fejer G, Wegner MD, Gyory I, Cohen I, Engelhard P, Voronov E, Manke T, Ruzsics Z, Dolken L, Prazeres da Costa O, Branzk N, Huber M, Prasse A, Schneider R, Apte RN, Galanos C, Freudenberg MA. 2013. Nontransformed, GM-CSF-dependent macrophage lines are a unique model to study tissue macrophage functions. Proc Natl Acad Sci U S A2013/05/28. 110:E2191-8.

31. Weischenfeldt J, Porse B. 2008. Bone marrow-derived macrophages (BMM): Isolation and applications. Cold Spring Harbor Protocols 3.

32. Fahy RJ, Exline MC, Gavrilin MA, Bhatt NY, Besecker BY, Sarkar A, Hollyfield JL, Duncan MD, Nagaraja HN, Knatz NL, Hall M, Wewers MD. 2008. Inflammasome mRNA expression in human monocytes during early septic shock. Am J Respir Crit Care Med2008/02/12. 177:983-988.

33. Carpenter AE, Jones TR, Lamprecht MR, Clarke C, Kang IH, Friman O, Guertin DA, Chang JH, Lindquist RA, Moffat J, Golland P, Sabatini DM. 2006. CellProfiler: image analysis software for identifying and quantifying cell phenotypes. Genome Biol2006/11/02. 7:R100.

34. Fulco CP, Munschauer M, Anyoha R, Munson G, Grossman SR, Perez EM, Kane M, Cleary $B$, Lander ES, Engreitz JM. 2016. Systematic mapping of functional enhancer-promoter connections with CRISPR interference. Science 354:769-773.

35. Shalem O, Sanjana NE, Hartenian E, Shi X, Scott DA, Mikkelsen TS, HeckI D, Ebert BL, Root DE, Doench JG, Zhang F. 2014. Genome-scale CRISPR-Cas9 knockout screening in human cells. Science (New York, NY) 343:84-87.

865

36. Doench JG, Fusi N, Sullender M, Hegde M, Vaimberg EW, Donovan KF, Smith I, Tothova Z, Wilen C, Orchard R, Virgin HW, Listgarten J, Root DE. 2016. Optimized sgRNA design to 
maximize activity and minimize off-target effects of CRISPR-Cas9. Nat Biotechnol2016/01/19. 34:184-191.

37. Chen S, Sanjana NE, Zheng K, Shalem O, Lee K, Shi X, Scott DA, Song J, Pan JQ, Weissleder $R$, Lee $H$, Zhang F, Sharp PA. 2015. Genome-wide CRISPR screen in a mouse model of tumor growth and metastasis. Cell 160:1246.

38. Huang DW, Sherman BT, Lempicki RA. 2009. Systematic and integrative analysis of large gene lists using DAVID bioinformatics resources. Nature protocols 4:44-57.

39. Maler MD, Nielsen PJ, Stichling N, Cohen I, Ruzsics Z, Wood C, Engelhard P, Suomalainen M, Gyory I, Huber M, Muller-Quernheim J, Schamel WWA, Gordon S, Jakob T, Martin SF, Jahnen-Dechent W, Greber UF, Freudenberg MA, Fejer G. 2017. Key role of the scavenger receptor MARCO in mediating adenovirus infection and subsequent innate responses of macrophages. mBio2017/08/03. 8.

40. Woo M, Wood C, Kwon D, Park KP, Fejer G, Delorme V. 2018. Mycobacterium tuberculosis infection and innate responses in a new model of lung alveolar macrophages. Front Immunol2018/03/30. 9:438.

41. Mould KJ, Jackson ND, Henson PM, Seibold M, Janssen WJ. 2019. Single cell RNA sequencing identifies unique inflammatory airspace macrophage subsets. JCI Insight2019/02/06. 4.

42. Pollard KM. 2016. Silica, silicosis, and autoimmunity. Frontiers in Immunology 7.

43. Parks CG, Miller FW, Pollard KM, Selmi C, Germolec D, Joyce K, Rose NR, Humble MC. 2014. Expert panel workshop consensus statement on the role of the environment in the development of autoimmune disease. Int J Mol Sci2014/09/10. 15:14269-14297.

44. Kuroda E, Ozasa K, Temizoz B, Ohata K, Koo CX, Kanuma T, Kusakabe T, Kobari S, Horie M, Morimoto Y, Nakajima S, Kabashima K, Ziegler SF, Iwakura Y, Ise W, Kurosaki T, Nagatake T, Kunisawa J, Takemura N, Uematsu S, Hayashi M, Aoshi T, Kobiyama K, Coban C, Ishii KJ. 2016. Inhaled fine particles induce alveolar macrophage death and interleukin-1alpha release to promote inducible bronchus-associated lymphoid tissue formation. Immunity2016/12/22. 45:1299-1310.

45. Rabolli V, Badissi AA, Devosse R, Uwambayinema F, Yakoub Y, Palmai-Pallag M, Lebrun A, de Gussem V, Couillin I, Ryffel B, Marbaix E, Lison D, Huaux F. 2014. The alarmin IL-1alpha is a master cytokine in acute lung inflammation induced by silica micro- and nanoparticles. Part Fibre Toxicol2014/12/17. 11:69.

46. Platt RJ, Chen S, Zhou Y, Yim MJ, Swiech L, Kempton HR, Dahlman JE, Parnas O, Eisenhaure TM, Jovanovic M, Graham DB, Jhunjhunwala $S$, Heidenreich $M$, Xavier RJ, Langer R, Anderson DG, Hacohen N, Regev A, Feng G, Sharp PA, Zhang F. 2014. CRISPRCas9 knockin mice for genome editing and cancer modeling. Cell 159:440-455.

47. Cortez JT, Montauti E, Shifrut E, Gatchalian J, Zhang Y, Shaked O, Xu Y, Roth TL, Simeonov DR, Zhang Y, Chen S, Li Z, Woo JM, Ho J, Vogel IA, Prator GY, Zhang B, Lee Y, Sun Z, Ifergan I, van Gool F, Hargreaves DC, Bluestone JA, Marson A, Fang D. 2020. CRISPR screen in regulatory T cells reveals modulators of Foxp3. Nature 582:416-420.

48. Thakur SA, Beamer CA, Migliaccio CT, Holian A. 2009. Critical role of MARCO in crystalline silica-induced pulmonary inflammation. Toxicol Sci2009/01/20. 108:462-471. 
908

909

910

911

912

913

914

915

916

917

918

919

920

921

922

923

924

925

926

927

928

929

930

931

932

933

934

935

936

937

938

939

940

941

942

943

944

945

946

947

948

949

950

951

49. Hornung V, Bauernfeind F, Halle A, Samstad EO, Kono H, Rock KL, Fitzgerald KA, Latz E. 2008. Silica crystals and aluminum salts activate the NALP3 inflammasome through phagosomal destabilization. Nat Immunol2008/07/08. 9:847-856.

50. Hamilton Jr. RF, Thakur SA, Mayfair JK, Holian A. 2006. MARCO mediates silica uptake and toxicity in alveolar macrophages from C57BL/6 mice. J Biol Chem2006/09/21. 281:34218-34226.

51. Kiritsy MC, Ankley LM, Trombley J, Huizinga GP, Lord AE, Orning P, Elling R, Fitzgerald KA, Olive AJ. 2021. A genetic screen in macrophages identifies new regulators of IFN $Y^{-}$ inducible MHCll that contribute to T cell activation. eLife 10.

52. Sanson KR, Hanna RE, Hegde M, Donovan KF, Strand C, Sullender ME, Vaimberg EW, Goodale A, Root DE, Piccioni F, Doench JG. 2018. Optimized libraries for CRISPR-Cas9 genetic screens with multiple modalities. Nature Communications 2018 9:1 9:1-15.

53. Li W, Xu H, Xiao T, Cong L, Love MI, Zhang F, Irizarry RA, Liu JS, Brown M, Liu XS. 2014. MAGeCK enables robust identification of essential genes from genome-scale CRISPR/Cas9 knockout screens. Genome biology 15:554.

54. Dupont S, Mamidi A, Cordenonsi M, Montagner M, Zacchigna L, Adorno M, Martello G, Stinchfield MJ, Soligo S, Morsut L, Inui M, Moro S, Modena N, Argenton F, Newfeld SJ, Piccolo S. 2009. FAM/USP9x, a deubiquitinating enzyme essential for TGFbeta signaling, controls Smad4 monoubiquitination. Cell 136:123-135.

55. Esteves F, Rueff J, Kranendonk M. 2021. The Central Role of Cytochrome P450 in Xenobiotic Metabolism-A Brief Review on a Fascinating Enzyme Family. Journal of xenobiotics 11:94-114.

56. Woods PS, Kimmig LM, Meliton AY, Sun KA, Tian Y, O'Leary EM, Gökalp GA, Hamanaka RB, Mutlu GM. 2020. Tissue-resident alveolar macrophages do not rely on glycolysis for LPS-induced inflammation. American Journal of Respiratory Cell and Molecular Biology 62:243-255.

57. Sinclair C, Bommakanti G, Gardinassi L, Loebbermann J, Johnson MJ, Hakimpour P, Hagan T, Benitez L, Todor A, Machiah D, Oriss T, Ray A, Bosinger S, Ravindran R, Li S, Pulendran B. 2017. mTOR regulates metabolic adaptation of APCs in the lung and controls the outcome of allergic inflammation. Science (New York, NY) 357:1014-1021.

58. Bissonnette EY, Lauzon-Joset JF, Debley JS, Ziegler SF. 2020. Cross-Talk Between Alveolar Macrophages and Lung Epithelial Cells is Essential to Maintain Lung Homeostasis. Front Immunol2020/11/13. 11:583042.

59. Fejer G, Sharma S, Gyory I. 2015. Self-renewing macrophages--a new line of enquiries in mononuclear phagocytes. Immunobiology2014/12/04. 220:169-174.

60. Luo M, Lai W, He Z, Wu L. 2021. Development of an Optimized Culture System for Generating Mouse Alveolar Macrophage-like Cells. The Journal of Immunology 207:1683-1693.

61. Gorki AD, Zymmank D, Zahalka S, Lakovits A, Hladik A, Langer B, Maurer B, Sexl V, Kain R, Knapp S. 2021. Murine ex vivo cultured alveolar macrophages provide a novel tool to study tissue-resident macrophage behavior and function. bioRxiv2021/02/11. https://doi.org/10.1101/2021.02.11.430791.

62. Kawasaki H. 2015. A mechanistic review of silica-induced inhalation toxicity. Inhalation Toxicology 27:363-377. 
63. Arredouani MS, Palecanda A, Koziel H, Huang YC, Imrich A, Sulahian TH, Ning YY, Yang Z, Pikkarainen T, Sankala M, Vargas SO, Takeya M, Tryggvason K, Kobzik L. 2005. MARCO is the major binding receptor for unopsonized particles and bacteria on human alveolar macrophages. J Immunol2005/10/21. 175:6058-6064.

64. Mao H, Kano G, Hudson SA, Brummet M, Zimmermann N, Zhu Z, Bochner BS. 2013. Mechanisms of Siglec-F-Induced Eosinophil Apoptosis: A Role for Caspases but Not for SHP-1, Src Kinases, NADPH Oxidase or Reactive Oxygen. PLOS ONE 8:e68143.

65. Feng $\mathrm{YH}$, Mao H. 2012. Expression and preliminary functional analysis of Siglec-F on mouse macrophages. Journal of Zhejiang University Science B 13:386.

66. Ginhoux F. 2014. Fate PPAR-titioning: PPAR-gamma "instructs" alveolar macrophage development. Nat Immunol2014/10/21. 15:1005-1007.

67. Coleman MM, Ruane D, Moran B, Dunne PJ, Keane J, Mills KHG. 2013. Alveolar macrophages contribute to respiratory tolerance by inducing FoxP3 expression in naive $T$ cells. American Journal of Respiratory Cell and Molecular Biology 48:773-780.

68. Li S, Lei Y, Lei J, Li H. 2021. All-trans retinoic acid promotes macrophage phagocytosis and decreases inflammation via inhibiting CD14/TLR4 in acute lung injury. Molecular medicine reports 24 .

69. Munger JS, Huang X, Kawakatsu H, Griffiths MJD, Dalton SL, Wu J, Pittet JF, Kaminski N, Garat C, Matthay MA, Rifkin DB, Sheppard D. 1999. A Mechanism for Regulating Pulmonary Inflammation and Fibrosis: The Integrin $\alpha \vee \beta 6$ Binds and Activates Latent TGF ß1. Cell 96:319-328.

70. Kannan S, Huang H, Seeger D, Audet A, Chen Y, Huang C, Gao H, Li S, Wu M. 2009. Alveolar epithelial type II cells activate alveolar macrophages and mitigate P. Aeruginosa infection. PloS one 4. 
A.

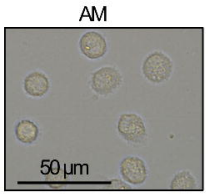

B.

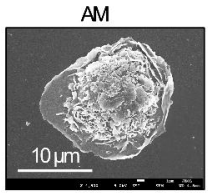

c.

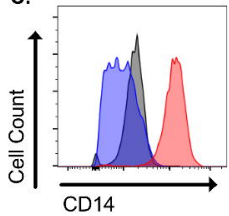

Fetal Liver Cells P2

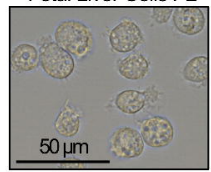

Fetal Liver Cells P2

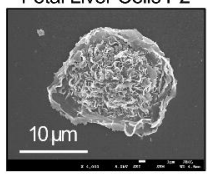

Fetal Liver Cells P14

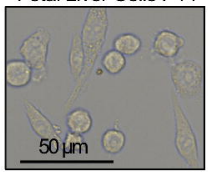

Fetal Liver Cells P17

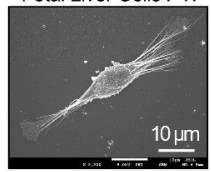

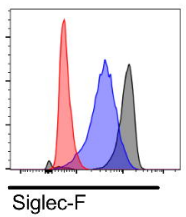

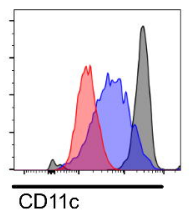

AMs

Fetal Liver

Cells P4

Fetal Liver

Cells P15

D.
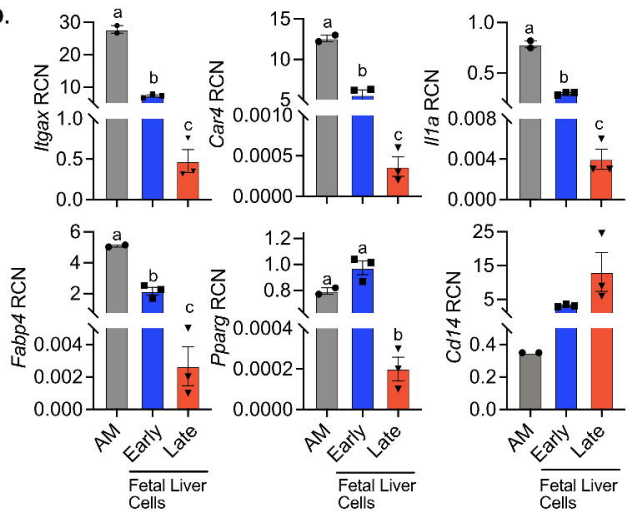

Figure 1 
A.

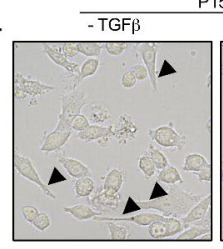

P15

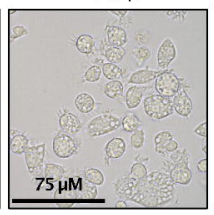

B.
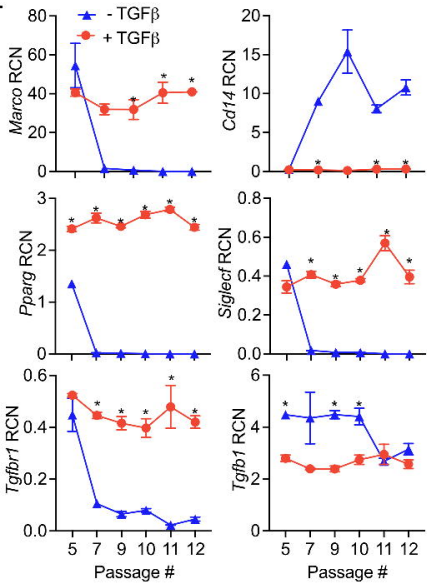

c.
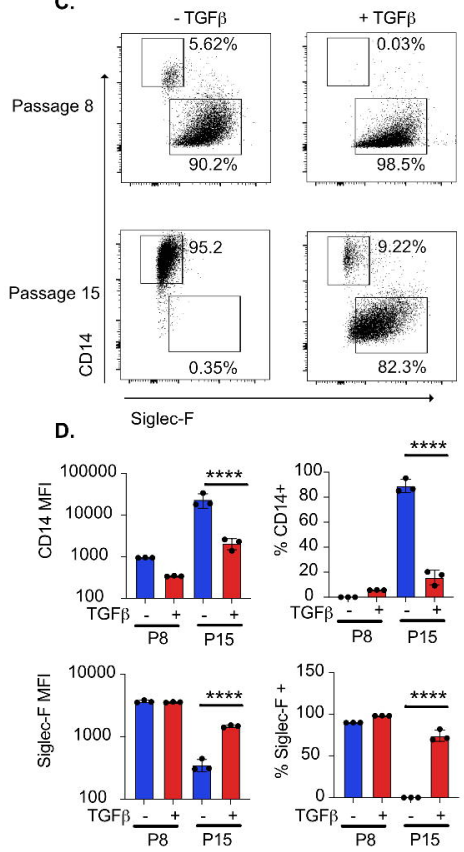

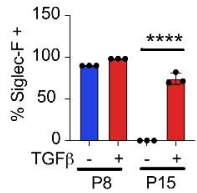

Figure 2 


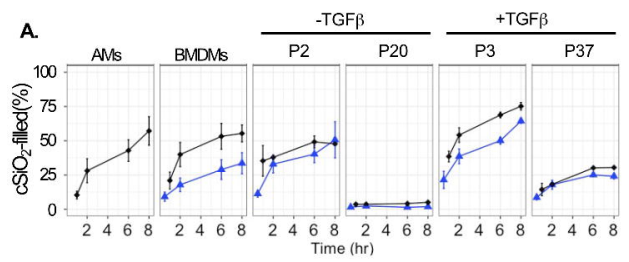

B.

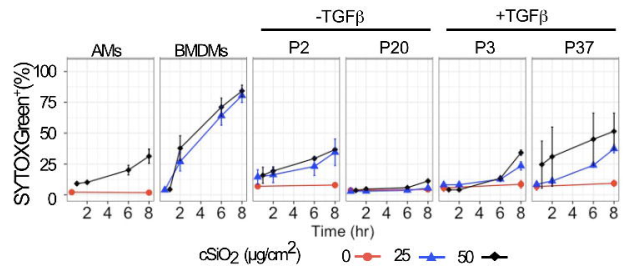

c.
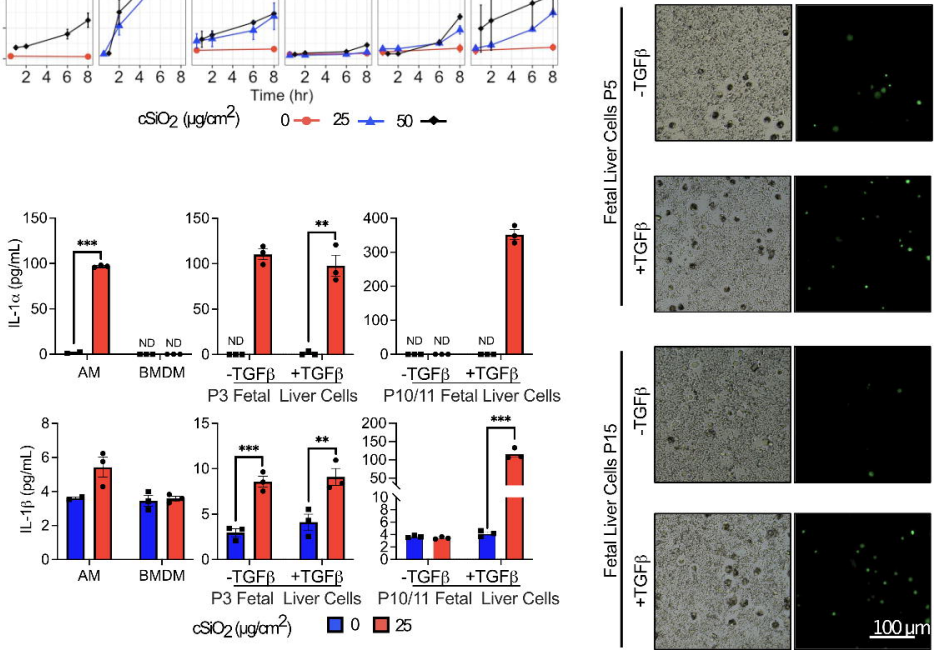

Figure 3 
$\square$ wild type $\square$ Marco KO $\square$ ॥1r KO

A.

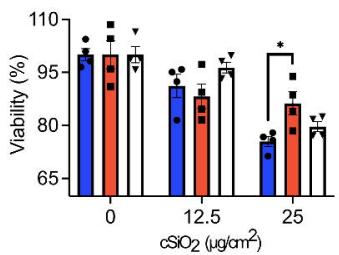

B.

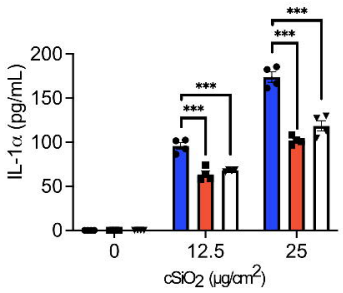

c.

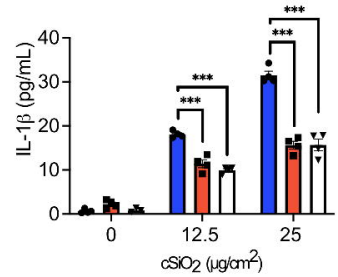

Figure 4 
A.

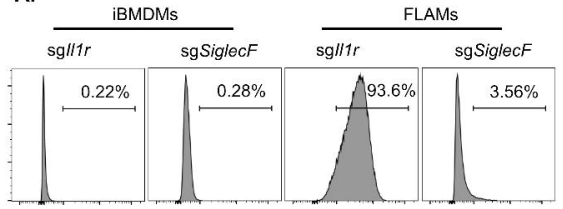

Siglec-F

c.

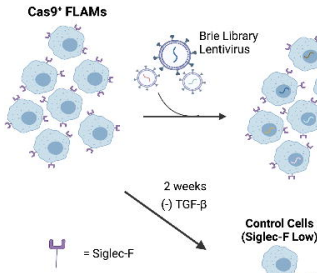

E.

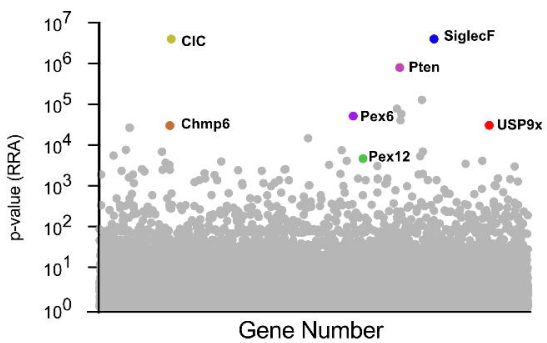

Knockout Library
B.

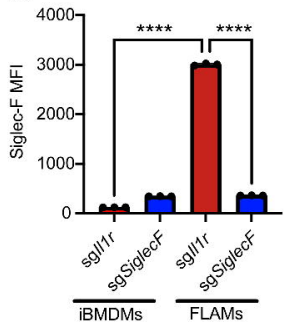

D.

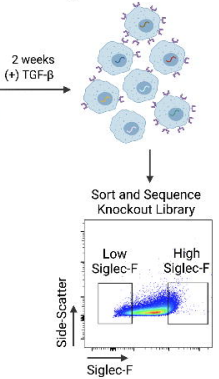

F.

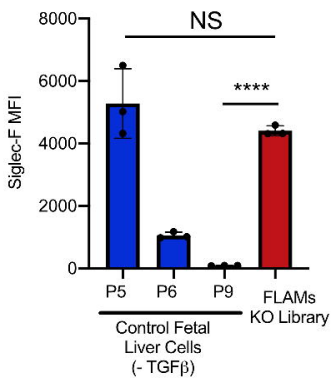

sgSiglecF

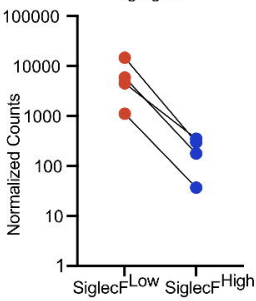

Figure 5 
A.

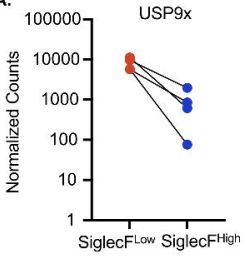

B.

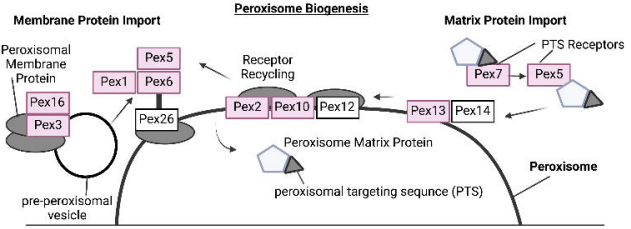

c.

Peroxisome Blogenesis

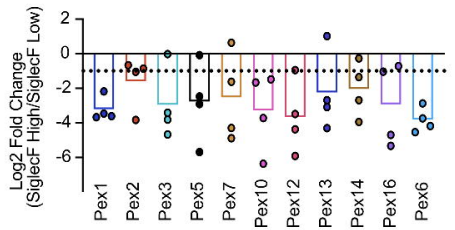

E.
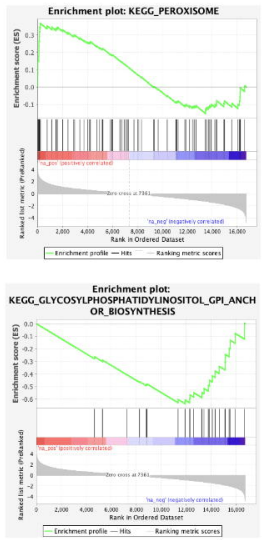

D.

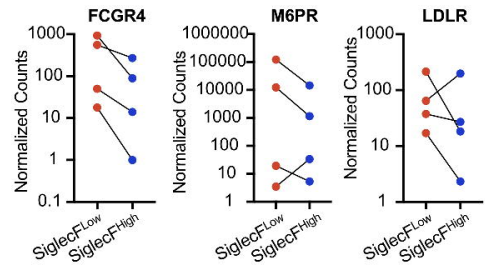

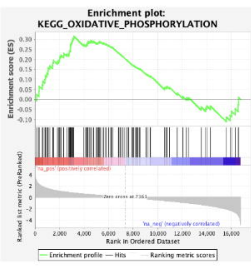

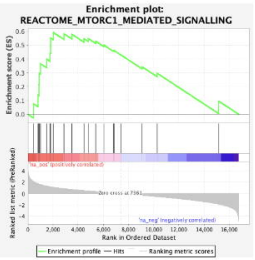

Figure 6 


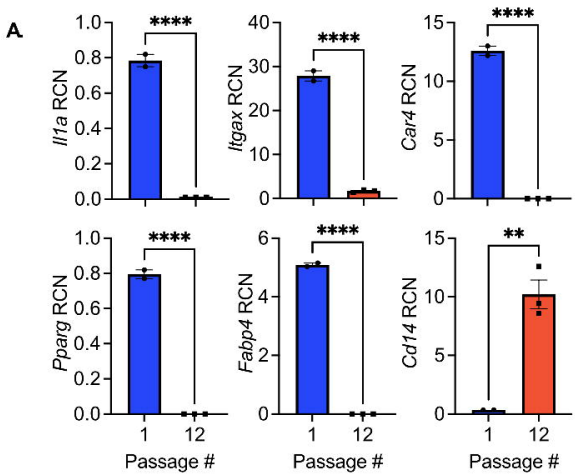

B.

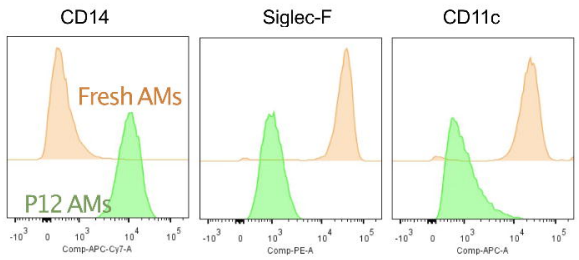

Figure S1 

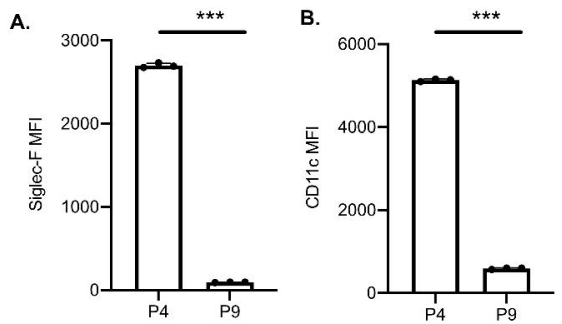

Figure S2 


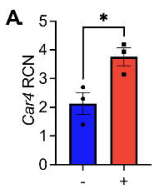

TGFbeta (10ng/mL)

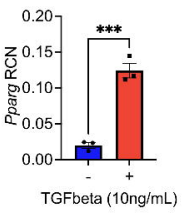

TGFbeta (10ng/mL)

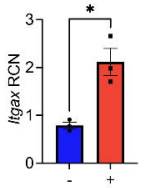

TGFbeta (10ng/mL)

B.
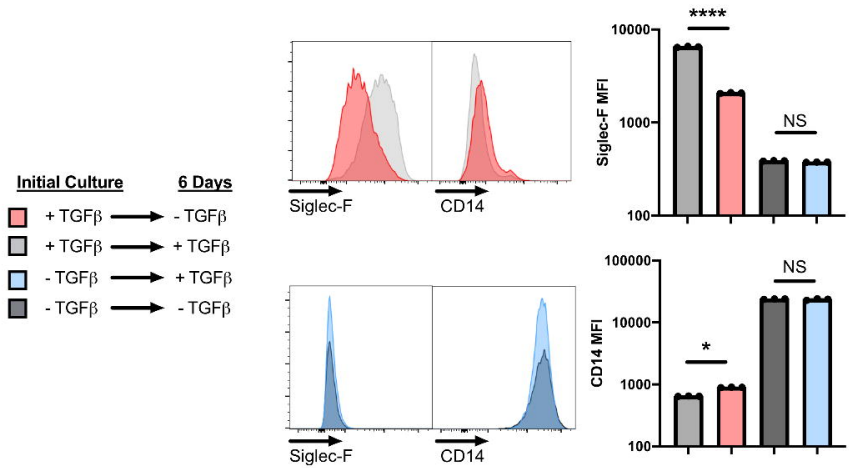

c.

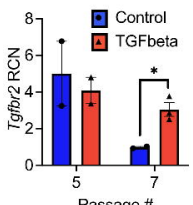

D.

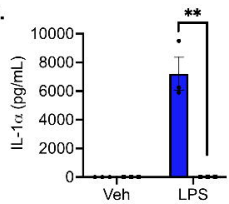

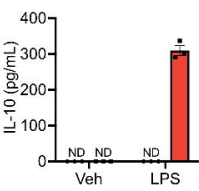

FLAMs

BMDM 
ndel Spectrum

A

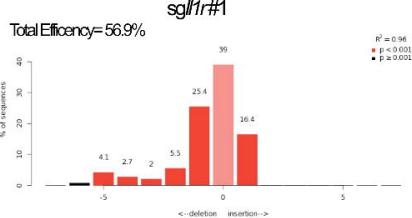

indel Spectrum

B. Total Efficency $=96.9 \%$ sgMaroo\#1

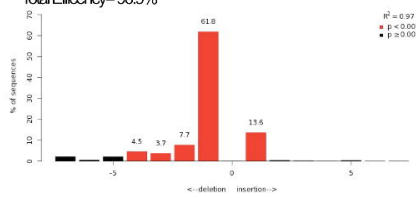

C.

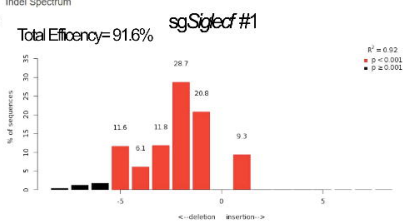

Inde1 Spectrum

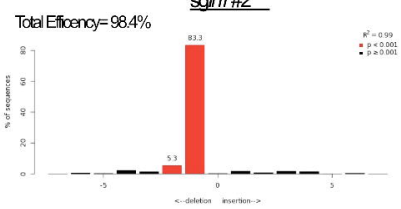

incel spectrum

Total Efficency $=89.5 \% \quad$ sgMarco\#2

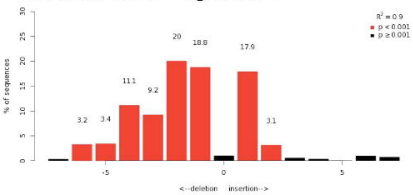

Incel Spectum

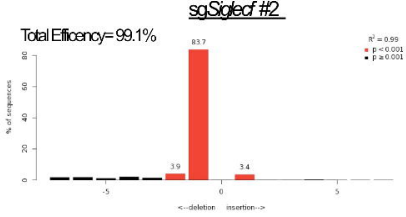

Figure S4 